\title{
Dichotic sequence discrimination: The effect of stimulus intensity and background noise level
}

\author{
HARVEY BABKOFF, DANIEL ALGOM, and SHLOMO ELIASHAR \\ Bar-Ilan University, Ramat-Gan, Israel
}

\begin{abstract}
Dichotic temporal discrimination of clicks over the range of separation of $2<\Delta t<96$ msec is nonmonotonic, being $V$ - or U-shaped with two maxima, one at very short separations ( $\Delta t<4 \mathrm{msec}$ ) and one at long separations $(\Delta t \geqslant 64 \mathrm{msec}$ ). Decreasing the level of the stimuli or increasing the level of background noise results in an overall decrement in discrimination level and a widening of the base of the function to make it more $U$-shaped. These effects are expressed in shorter $\Delta t$ thresholds for the left limb of the function and longer $\Delta t$ thresholds for the right limb of the function.
\end{abstract}

At least two fundamentally different types of discrimination occur with the separation of two dichotic stimuli over the temporal range of 2 to $96 \mathrm{msec}$, and both are reflected in the psychophysical data (Babkoff, 1975; Babkoff, Algom, \& Eliashar, 1980). When subjects are required to discriminate between pairs of clicks presented to the two ears and separated by an interval extending from 2 to $96 \mathrm{msec}$, a bisegmented, nonmonotonic, $\mathrm{V}$ - or U-shaped function results (Babkoff, 1975; Babkoff, Algom, \& Eliashar, 1980). Discrimination level is high for dichotic temporal separations $(\Delta t)$ of 2 to $4 \mathrm{msec}$, decreases with $6<\Delta \mathrm{t} \leqslant 16 \mathrm{msec}$, and increases from $16-24 \mathrm{msec}$ to $96 \mathrm{msec}$. The left descending limb of the curve is assumed to reflect the "breakup" of the fused stimulus (at very short $\Delta$ ts), while the right segment is assumed to reflect temporal order discrimination, increasing with $\Delta t>16-24$ msec (Babkoff et al., 1980).

The recent literature on the discrimination of monaural and diotic sequences indicates that temporal masking and stimulus level may affect the perception of their temporal order (Divenyi \& Danner, 1977; Divenyi \& Hirsh, 1975; Homick, Elfner, \& Bothe, 1969).

Homick et al. (1969) studied the effects of intensity on the perception of temporal order and temporal masking of one of the stimuli. Their results indicate that temporal masking is greater and the perception of temporal order is more difficult for a lowlevel stimulus than for a higher level stimulus. These

Dr. Babkoff is spending the $1980-1981$ academic year at Walter Reed Army Institute of Research, Department of Military Medical Psychophysiology, Forest Glen Annex, Building 189, Washington, D.C. 20012. The experiments were conducted at the Bar-Ilan University, Ramat-Gan, Israel. The authors would like to thank the Bar-Ilan Research Committee for making funds avaliable to conduct the experiments. authors note that their data support the hypothesis of Hirsh (1959) of a hierarchy of perceptions associated with the temporal separation of stimuli. They also note, however, that the congruence of results of a monaural masking paradigm and a monaural temporal order paradigm may reflect the limitations imposed solely by peripheral stimulus coding mechanisms.

An additional test of the concept of a hierarchy of levels of perception that includes central as well as peripheral mechanisms would be that of dichotic stimuli separated in time. Since such stimuli do not interact at neural auditory levels lower than brainstem structures (e.g., the superior olives), a study of the effects of manipulating intensity and background noise level on dichotic sequence discrimination might provide additional information on the function of more central mechanisms and help identify an appropriate theoretical framework for such types of discrimination. No data are available regarding the effect of manipulating these parameters on dichotic two-pulse sequence discrimination. The present study consists of two experiments designed to study the effects of manipulating stimulus intensity and background noise level on the discrimination of a pair of dichotic clicks.

\section{EXPERIMENT 1}

\section{Apparatus and Method}

The interval between the dichotic clicks ( $\Delta t$ ) was varied from 2 to 96 msec. All $\Delta$ ts were generated by a crystal-controlled timer (local design). Intra- and intertrial intervals were controlled by Massey-Dickinson logic units and by locally designed circuitry. All $\Delta$ ts and durations were calibrated and monitored with a Monsanto Type 120A Counter-Timer. Error did not exceed $.05 \%$.

The transient stimuli were generated as square waves and shaped as negative-going pulses with an exponential return to base $\left(e^{-1}=\right.$ $.1 \mathrm{msec})$. The negative-going exponential pulses were transduced 
as clicks by a set of AKC K60 stereo earphones. The click is an initial rarefaction pulse with ringing and slight condensation overshoot before return to base.

A three-alternative forced-choice design ${ }^{1}$ was used (Babkoff et al., 1981). The subject was seated in a Medtechnic Silent Cabin facing a panel with a warning light, a feedback light, and three response keys (marked 1, 2, and 3). A trial lasted $4 \mathrm{sec}$. Intertrial intervals were approximately $6 \mathrm{sec}$, during which the subject responded.

Eight $\Delta$ ts were tested: $2,4,6,16,24,48,64$, and $96 \mathrm{msec}$. On any given trial, $\Delta t$ was the same for the three presentation intervals (see Footnote 1). $\Delta$ t was randomized by trial. Each $\Delta t$ was presented 100 times, so that a dichotic discrimination function is based on $\mathbf{8 0 0}$ trials for each subject for each click sensation level.

In the first experiment, a dichotic discrimination was studied at three different click levels: 10,20 , and $35 \mathrm{~dB} \mathrm{SL}$. Monaural thresholds of each subject were measured before the experiment by the method of constant stimuli and were monitored throughout the experiment. Since no large changes in threshold were recorded, these estimates were maintained throughout and served as the referents for determining the click sensation levels for each subject. Intensity level was fixed for a given experimental session and was randomized across sessions.

Each subject was tested over 24 sessions of 100 trials each. Subjects received feedback after each trial as to the correctness of the response. Prior to data collection, subjects were trained for three sessions on all $\Delta$ ts and intensity levels subsequently used in Experiment 1. Six subjects participated in this experiment.

\section{Results}

A two-way analysis of variance for repeated measurements was performed on the discrimination scores of each subject at each $\Delta t$ and intensity level. Both of the main effects are significant $[\Delta t: F(7,35)$ $=31.89, \mathrm{p} \leqslant .0001$; intensity: $\mathrm{F}(2,10)=4.83, \mathrm{p} \leqslant .03]$. The interaction term is not significant.

The average discrimination level data for the six subjects are plotted on the ordinate of Figure 1 as a

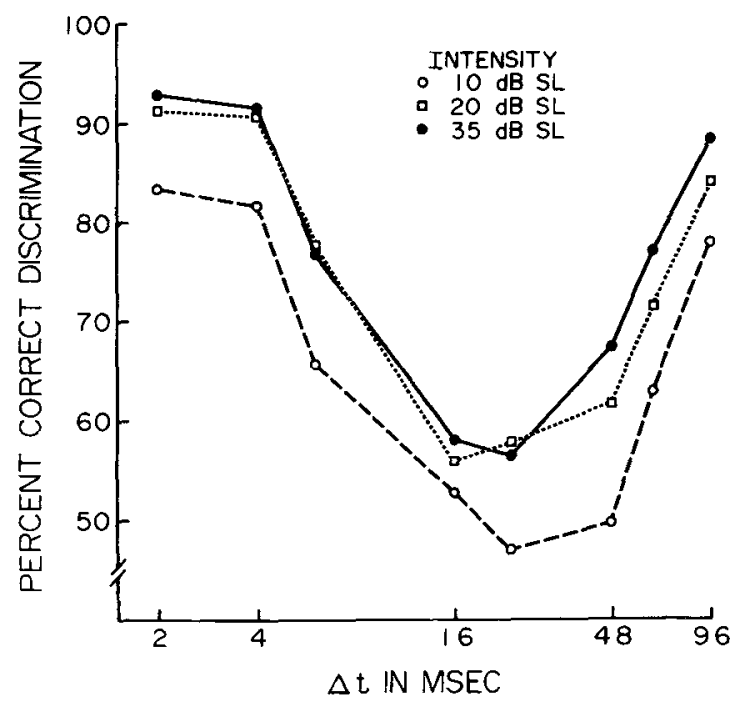

Figure 1. Percent correct discrimination is plotted on the ordinate as a function of the temporal separation $(\Delta t)$ of the dichotic clicks on a logarithmic abscissa. Data are shown for three click sensation levels.

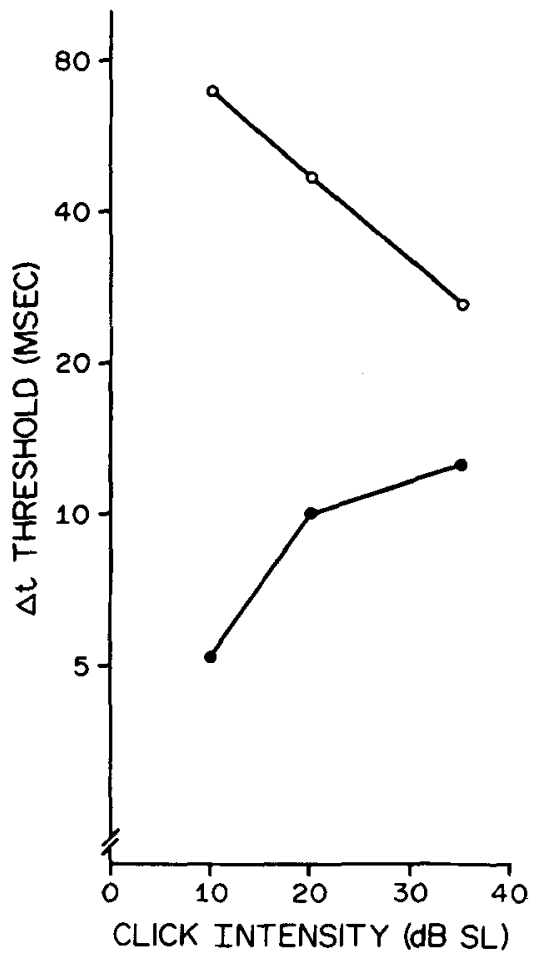

Figure 2. Dichotic discrimination threshold in milliseconds is plotted on a logarithmic ordinate as a function of click sensation level in decibels (SL) on the abscissa. The lower curve represents the left-limb threshold (Figure 1), while the upper curve represents the right-limb threshold (Figure 1).

function of interaural time asymmetry $(\Delta t)$ on a logarithmic abscissa. The parameter of the curves is click sensation level. The functions are nonmonotonic, V-, or U-shaped. As click sensation level is decreased from 35 to $10 \mathrm{~dB}$, discrimination level decreases at all $\Delta$ ts. In addition, there appears to be a widening of the base of the curve to appear more $U$ than V-shaped.

Subsequent analyses were performed based on the assumption that two psychometric mechanisms of opposite sign are contributing to the V-shape of the function. The data of each subject were subdivided into two groups ( $2 \mathrm{msec} \leqslant \Delta \mathrm{t} \leqslant 16 \mathrm{msec}$ and $24 \mathrm{msec}$ $\leqslant \Delta \mathrm{t} \leqslant 96 \mathrm{msec}$ ) and were analyzed separately by probit and by least squares analyses. The descending and ascending functions can be fitted by cumulative Gaussian distributions. Of 36 functions, $95 \%$ of the variance is accounted for in 18 functions and $75 \%$ of the variance is accounted for in 14 functions.

$\Delta t$ thresholds ${ }^{2}$ for the descending and ascending limbs of the functions were estimated for each subject at the three click sensation levels and were analyzed separately by one-way analyses of variance for repeated measurements. The results indicate that both $\Delta t$ thresholds are significantly affected by increases in click sensation level $(\mathrm{p} \leqslant .05)$. The average $\Delta t$ thresholds are plotted in Figure 2 in milliseconds 
on a logarithmic ordinate as a function of click sensation level in decibels on the abscissa. The average threshold of the left limb of the functions increases from 5.23 to $12.45 \mathrm{msec}$ as click sensation level increases from 10 to $35 \mathrm{~dB}$. The average threshold of the right limb of the functions decreases from 69.6 to $26 \mathrm{msec}$ as click sensation level increases from 10 to $35 \mathrm{~dB}$. Note the almost-mirror-image divergence of the two curves representing the left- and right-limb thresholds as click sensation level is decreased from 35 to $10 \mathrm{~dB}$.

\section{EXPERIMENT 2}

The effect of background noise level on dichotic discrimination was studied, using the same general experimental design as in Experiment 1 (see Footnote 2).

Dichotic discrimination functions for a pair of 35dB-SL clicks were generated at three levels of background, binaurally presented, coherent (same noise generator) broadband noise: 0,20 , and $40 \mathrm{~dB}$ SL. Monaural noise thresholds were determined by the method of constant stimuli and served as the referents for each subject for the three noise levels. Noise level was fixed within each session and was randomized across sessions. Four trained subjects who had not participated in Experiment 1 participated in this experiment.

\section{Results}

A two-way analysis of variance for repeated measurements was performed on the discrimination scores of each subject at each $\Delta t$ and background noise level. Both of the main effects are significant $[\Delta t: F=(7,21)=9.43, p \leqslant .00005$; noise level: $F(2,6)$ $=48.44, \mathrm{p} \leqslant .0002]$. The interaction term is not significant.

The average discrimination level data for the four subjects are plotted on the ordinate of Figure 3 as a function of $\Delta t$ on a logarithmic abscissa. The parameter of the curves is background noise level. Note, as in Figure 1, all the functions are nonmonotonic, V-, or U-shaped. As the sensation level of background noise is increased from 0 to $40 \mathrm{~dB}$, there is an overall decrease in discrimination level at all $\Delta t s$, resulting in a clear downward displacement along the ordinate of the curve representing 35-dBSL clicks presented against a background noise sensation level of $\mathbf{4 0} \mathrm{dB}$. In addition, there is a clear widening of the base of the curve to appear U-shaped rather than V-shaped.

As in Experiment 1, the data of each subject were subdivided into two groups $(2 \mathrm{msec} \leqslant \Delta \mathrm{t} \leqslant 16 \mathrm{msec}$ and $24 \mathrm{msec} \leqslant \Delta \mathrm{t} \leqslant 96 \mathrm{msec}$ ) and were analyzed separately by probit and by least squares analyses. The descending and ascending functions can be fitted

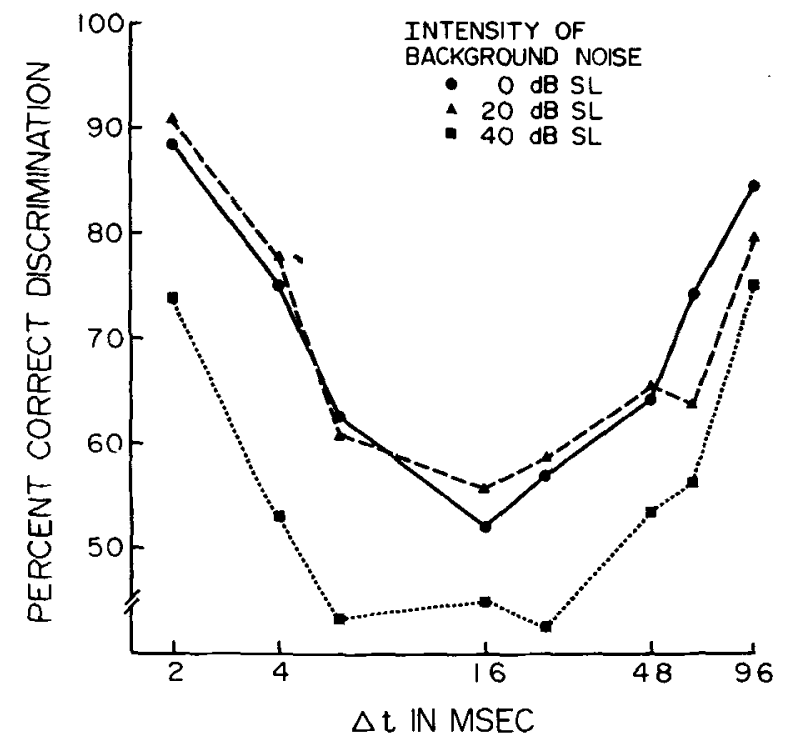

Figure 3. Percent correct discrimination is plotted on the ordinate as a function of the temporal separation ( $\Delta t)$ of the dichotic clicks on a logarithmic abscissa. Click sensation level is $35 \mathrm{~dB}$. Data are shown for three background noise sensation levels.

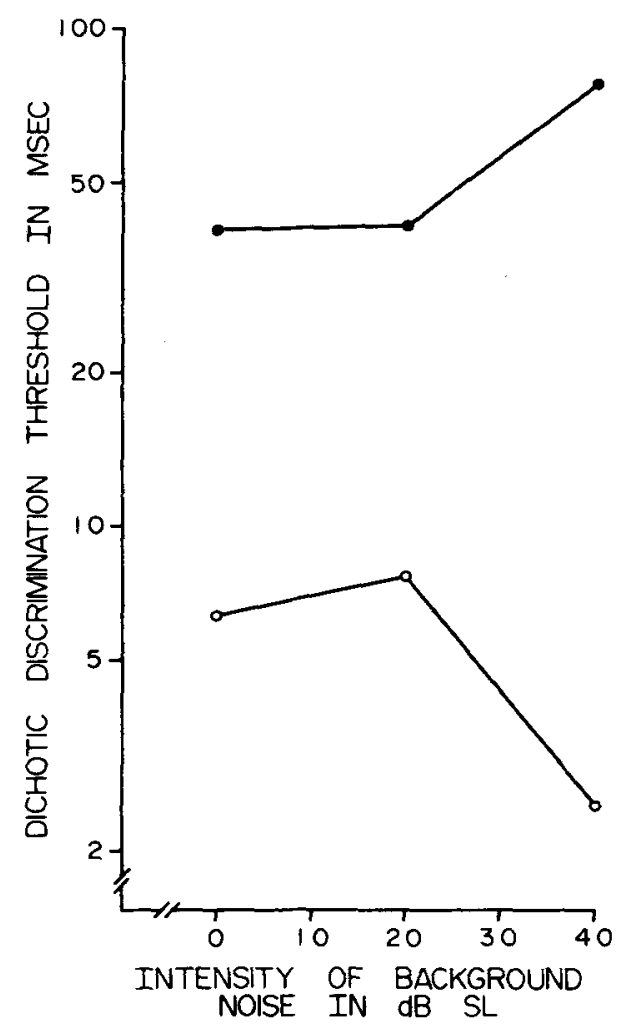

Figure 4. Dichotic discrimination threshold in milliseconds is plotted on a logarithmic ordinate as function of the intensity of background noise in decibels (SL) on the abscissa. The lower curve represents the left-limb threshold (Figure 2), while the upper curve represents the right-limb threshold (Figure 2). 
by cumulative Gaussian distributions, although these fittings account for less of the overall variance of the functions in Experiment 2 than in Experiment 1. Of the 24 functions, $95 \%$ of the variance is accounted for in 6 functions and $75 \%$ of the variance is accounted for in 17 functions. $^{3}$

$\Delta t$ thresholds (see Footnote 2) for each of the two limbs of the function were estimated for each subject at the three background noise levels and were analyzed separately by one-way analyses of variance for repeated measurements. The results indicate that both thresholds are significantly affected by increases in noise level $(p \leqslant .05)$. The average $\Delta t$ thresholds are plotted in Figure 4 in milliseconds on a logarithmic ordinate as a function of background noise sensation level in decibels on the abscissa. The average threshold of the left limb of the functions decreases from 6.3 to $2.6 \mathrm{msec}$ as background noise level increases from 0 to $40 \mathrm{~dB} \mathrm{SL}$ (see Footnote 3). The average threshold of the right limb of the function increases from 40.8 to $84.1 \mathrm{msec}$ as background noise level increases from 0 to $40 \mathrm{~dB}$ SL. Note also the almostmirror-image divergence of the two curves representing the left- and right-limb thresholds as background noise sensation level is increased from 0 to $40 \mathrm{~dB}$.

In summary, a decrease in click sensation level or an increase in background noise level results in a downward displacement and widening of the nonmonotonic, V-shaped, dichotic discrimination function, which is expressed in a shorter $\Delta t$ threshold for the left limb and a longer $\Delta t$ threshold for the right limb. The effects of increasing background noise level on dichotic sequence discrimination are very similar to those achieved by decreasing the level of the stimuli.

\section{DISCUSSION}

The results of both experiments support our previous findings (Babkoff, 1975; Babkoff et al., 1980) that dichotic sequence discrimination is nonmonotonic over the range of interaural time separations $(\Delta t)$ from 2 to $96 \mathrm{msec}$, with anchor points of high discrimination at the shortest and longest $\Delta$ ts.

The discrimination of temporal order or of the sequence of monaural stimuli is adversely affected by a decrease in the intensity of the stimuli or by masking one or more of the stimuli (Divenyi \& Danner, 1977; Homick et al., 1969). The results of the present experiment extend this conclusion to the discrimination of dichotic two-pulse sequences as well. Decreasing the level of the stimuli or increasing the level of the background noise adversely affects discrimination of both limbs of the V-shaped function in an equivalent manner. This is expressed by: (1) an approximately equivalent decrease in discrimination level at all $\Delta t s ;$ and (2) an increasing separation of the two limbs of the curve to produce more of a Urather than a V-shaped function. This latter point is emphasized by noting the nearly mirror-image divergence of the two curves that represent the $\Delta t$ thresholds for the left and right limbs as stimulus intensity decreases or as the level of the background noise increases.

Given that the two limbs of the dichotic two-pulse sequence discrimination function represent different perceptual phenomena (Babkoff, 1975, 1980; Babkoff \& Sutton, 1963; Sternberg \& Knoll, 1973), the finding that decreasing intensity level and increasing background noise level have almost equivalent effects on the two limbs of the function is interesting. This might imply that these manipulations not only affect the specific characteristics of the stimuli per se (such as their loudness, timbre, etc.), but also affect the informational dimension necessary for the mechanisms involved in both types of discrimination to operate at maximum efficiency. What is common to both mechanisms operating on pairs of stimuli dichotic with respect to time is the time scale separating the stimuli when they are considered as generators of neural signals distributed along a temporal continuum. If both mechanisms associated with the two limbs of the function use neural signals distributed along a time scale and separated from each other but operate merely on different ranges of the scale, then a spread in the distribution of the neural signals caused by a decrease in level or an increase in background noise might serve to increase overlap in the distributions, decrease their separation, and degrade performance equally at all points along the scale. These results emphasize the time-marking aspect of the two dichotic stimuli, even when perceived as single localizable stimuli with very short interstimulus separations, rather than the interstimulus "relation" of the stimuli to each other (Sternberg \& Knoll, 1973).

\section{REFERENCES}

BAвкoFf, H. Dichotic temporal interactions: Fusion and temporal order. Perception \& Psychophysics, 1975, 18, 267-272.

BabKoff, H. Dichotic temporal interactions: Nonmonotonic discrimination function. Perception \& Psychophysics, 1980, 27, 273-275.

Babkoff, H., Algom, D., \& Eliashar, S. Dichotic sequence discrimination: The effect of continued practice. Psychological Research, 1980, 42, 319-326.

Babkoff, H., \& Sutton, S. Perception of temporal order and loudness judgments for dichotic clicks. Journal of the Acoustical Society of America, 1963, 35, 574-577.

Divenyi, P. L., \& DANner, W. F. Discrimination of time intervals marked by brief acoustic pulses of various intensities and spectra. Perception \& Psychophysics, 1977, 21, 125-152.

Divenyi, P. O., \& Hinsh, I. J. The effect of blanking on the identification of temporal order in three-tone sequences. Perception \& Psychophysics, 1975, 17, 246-252.

Hinsh, I. J. Auditory perception of temporal order. Journal of the Acoustical Society of America, 1959, 31, 759-767. 
Homick, J. L., Elffner, L. F., \& Bothe, G. G. Auditory temporal masking and the perception of order. Journal of the Acoustical Society of America, 1969, 45, 712-718.

Sternberg, S., \& Knoll, R. L. The perception of temporal order: Fundamental issues and a general model. In S. Kornblum (Ed.), Attention and performance IV. New York: Academic Press, 1973.

\section{NOTES}

1. A trial was signaled by the onset of a warning light and consisted of three 1-sec presentation intervals. In two of the intervals ("same"), a single pair of dichotic clicks was presented with either the first click to the right ear and the second click

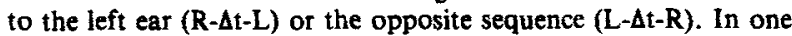
of the intervals ("different"), the order of the single pair of dichotic clicks was opposite to that of the other two intervals. The "different" pair was thus the dichotic "mirror image" of the "same" pairs. The subject's task was to listen to the three presentation intervals during a trial and to respond by depressing the key $(1,2$, or 3$)$ corresponding to the presentation interval containing the "different" stimulus (i.e., to discriminate the "different" from the "same" presentations).

2. The $\Delta t$ corresponding to the point at which the fitted $Z$ score $/ \log \Delta t$ curve crossed the $Z=.44$ ordinate $(p=67 \%$, appropriate for a three-alternative forced-choice procedure) was used as the best-fitting estimate of threshold for each subject for each condition.

3. The overall intra- and intersubject variance in Experiment 2 was greater than in Experiment 1, even for the comparable 35 $\mathrm{dB}-\mathrm{SL}$, background noise level $=0$-dB-SL condition. Consequently, the estimates of threshold of both limbs of the function differed across the two experiments. However, despite these baseline differences, the shapes of the dichotic discrimination functions as well as the effects of the parametric manipulations, decreasing click sensation level or increasing background noise level, produced the same overall effect on the V-shaped functions for all subjects in both experiments.

(Manuscript received March 30, 1981; accepted for publication May 7, 1981.) 